\title{
Interactive Multimedia Contents in the IllusionHole
}

\author{
Tokuo Yamaguchi, Kazuhiro Asai, \\ Yoshifumi Kitamura, and Fumio Kishino \\ Graduate School of Information Science and Technology, Osaka University, \\ 2-1 Yamada-oka, Suita, Osaka 565-0871, Japan \\ \{yamaguchi.tokuo, asai, kitamura,kishino\}@ist.osaka-u.ac.jp
}

\begin{abstract}
This paper proposes a system of interactive multimedia contents that allows multiple users to participate in a face-to-face manner and share the same time and space. It provides an interactive environment where multiple users can see and manipulate stereoscopic animation with individual sound. Two application examples are implemented; one is location-based content design and the other is user-based content design. Both effectively use a unique feature of the IllusionHole, i.e., a location-sensitive display device that provides a stereoscopic image with multiple users around the table.
\end{abstract}

Keywords: 3D user interface, entertainment computing, tabletop display, interactive, CSCW, game, stereoscopic display.

\section{Introduction}

Interactive multimedia contents combined with images and sounds have become widespread in various fields. These contents allow for abundant expression by users. For example, using virtual reality technologies, we can enjoy rich high-immersion experiences using multimodal interactions with various information channels such as visual, auditory, or tactile perception. Moreover, we can easily communicate at remote locations through networks and simultaneously share images and sounds in a huge virtual environment. There are, however, some limitations; for example, each of multiple users will not be able to get such rich high-immersion experiences simultaneously because communication channels through the network are not sufficient to convey non-verbal information that is important for natural communications.

In order to achieve natural interactions or collaborations for multiple users, tabletop approaches have been focused on. These approaches can provide a common workspace where multiple users may interact with each other and maintain awareness of what the others are doing. Based on this idea, many researchers explore the role of novel interfaces and interaction paradigms in the context of applications for entertainment. There is, however, little reported in the literature about tabletop displays that allow multiple users to enjoy stereoscopic animation with individual sound.

In this paper, we propose a multimedia content display system for co-located multiple users with which they can enjoy interactive multimedia content (such as a game) or work cooperatively through natural face-to-face communications while sharing the same time and space. The system is based on the IllusionHole [7]. It provides an 
interactive environment where multiple users can see and manipulate stereoscopic animation with individual sound. The content is basically common to all users; however, it is slightly varied or personalized, such as direction of animations or volume of sounds, according to the user's interactions or dynamically changing positional relationships with other users. Two types of application examples are implemented. Both provide individual animation and sound with a corresponding user, as well as the location-based content design and the user-based content design.

\section{Related Work}

Recently, some studies have shown that the combination of visual and auditory cues enhances the sense of immersion in virtual reality or interactive entertainment applications. This section outlines a variety of interactive stereoscopic displays and interfaces using auditory feedback.

To view objects from different angles by moving one's head provides humans with important clues for spatial cognition. One of the most reasonable ways to create a multi-user interactive stereoscopic display is to install a horizontal screen in a table [1]. This is the most effective way to view stereoscopic images from the vantage point of individuals standing around a table. And to use optical equipment that includes a parallax barrier [4], mirror [2,11] and revolving screen [5] allows multiple users to observe the stereoscopic images with motion parallax in any direction. In addition, multiple users are able to directly point to a particular part of the stereoscopic images in the IllusionHole [7].

Many co-located collaborative applications often present information to users through auditory channels as well as through visual feedback. For providing awareness of the users' actions, large-screen tiled displays use auditory information when users perform gestures or move objects [10]. Morris et al. found that increasing collaboration can result when individual, rather than public, auditory feedback is provided [9]. For music tabletops, the reacTable uses physical objects to represent parts of a modular synthesizer [6], and the Audiopad is a composition and performance instrument for electronic music that tracks the positions of objects on a tabletop [12]. Jam-O-Drum allows users to collaboratively create music around a circular tabletop [3], and with Multi-Audible [8], multiple users have a portable device to hear different audio information during the interaction.

\section{System Configuration}

In this section, we detail the configuration of an interactive multimedia content system that allows multiple users to see and manipulate stereoscopic animation with individual sound.

\subsection{Overview}

Our proposed multimedia content system allows multiple users standing around a table to interact with position-specific stereoscopic animation with individual sound. The content itself is basically common to all users; however, it is slightly varied or 
personalized according to users' interactions. We have implemented two different types of content design frameworks; i.e., location-based content design and userbased content design. In the former framework, stereoscopic animation and sound changes interactively according to the location where a user stands. The environment around the table is divided into an adequate number of domains. If a user comes into one domain from another, the content that is currently displayed to the user changes according to the domain. On the other hand, in the user-based content design, the multimedia content changes according to the user's positional relationships with other users. In this case, each user is also provided with interactive animation and sound, and they are changed when the surrounding environment changes, such as when another user comes closer or moves away.

\subsection{Implementation}

A prototype system was established using the IllusionHole with polarization filters (see [7] for more details). Based on the IllusionHole, the prototype system allows multiple users to see interactive stereoscopic animations with adequate motion parallax. In addition, Bluetooth-enabled wireless headphones are installed to provide individual sound for corresponding users. The system configuration is shown in Figure 1. The viewing position of each user is

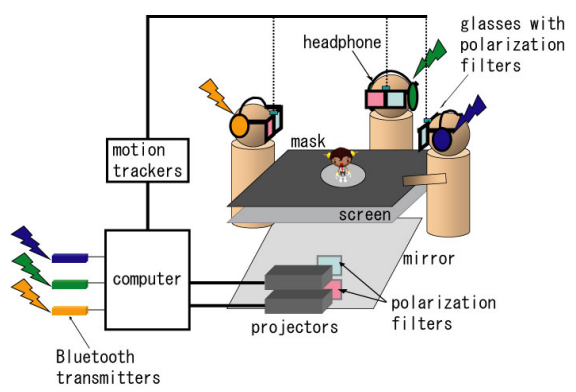

Fig. 1. System configuration detected using a 3D tracker device (IS-600

Mark 2 ultrasonic beacon, made by Intersense) and stereoscopic images are displayed using parallax images for both eyes, which are calculated for corresponding display regions. The display regions of multiple users may overlap each other if the number of users increases and neighboring users stand too close to one another. So, the prototype system is designed for three users. Each user wears a pair of circularly polarized glasses and a wireless headphone corresponding to an output sound channel.

A direct graphics library is used to manage and show stereoscopic animations of $3 \mathrm{D}$ characters. In addition, a direct sound library is used for generating multi-channel sound for individual sound. Figure 2 shows the output flow of individual sound. The direct sound library supports multiple buffers of multi-channel sound, and also supports the mixing of multiple sound buffers to play these sounds. Multiple buffers are assigned to each individual user, and these buffers have some slots for writing sound data which output from each channel (ch1 to ch8). The first slot " $\mathrm{H}$ " includes a header for the buffer. The sound data for only a particular user is written at the output channel assigned to her/him (e.g., user $\mathrm{A}$ is assigned $\operatorname{ch} 1$ and $\operatorname{ch} 4$, and user $\mathrm{C}$ is assigned ch3 and ch6), and null data is written in the remaining channel for silent output. By mixing the buffers in which sound data is written for each user, and controlling sound volume and the starting or stopping of sound, we manage the individual sounds. 

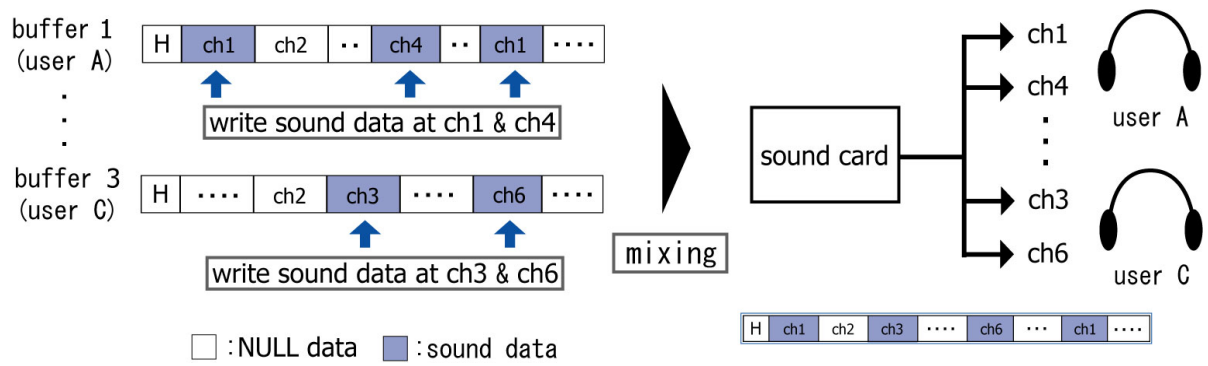

Fig. 2. Output flow of individual sound

\section{Application Examples}

We introduce two application examples that allow multiple users to communicate interactively in the same place at the same time using this configuration. "Onstage Demo of the IllusionHole" is an example of the location-based content design, and "Baa Baa White Sheep" is an example of the user-based content design.

\subsection{Onstage Demo of the IllusionHole}

In this application, the character shown at the center of the IllusionHole talks about functions and features of the IllusionHole itself. Figure 3 shows a snapshot of this application experienced by three users and different stereoscopic animations observed by individual users standing to the left, center, and right of the IllusionHole. The particular moment of Figure 3 shows that the 3D character faces to the left; therefore, only the left user (user B) hears the individual announcement as "Raise your hand"

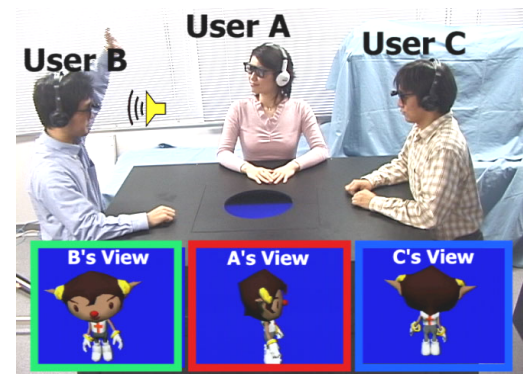

Fig. 3. Snapshot of Onstage Demo of the IllusionHole experienced by three users by the character; however, the other users (users A and C) cannot hear anything. In this application example, the character turns to other directions and talks about the IllusionHole similarly. The IllusionHole is a location-sensitive display device that provides a stereoscopic image with multiple users around the table, and each of the users can observe the same virtual object from a different direction. Therefore, this application example effectively uses this feature of the IllusionHole.

\subsection{Baa Baa White Sheep}

This application is designed so that a character in the IllusionHole corresponds to a user's actual position and movements in the physical world. A snapshot of users enjoying this application is shown in Figure 4. By simply moving around the display, each user can manipulate his/her own character without using devices such as game 


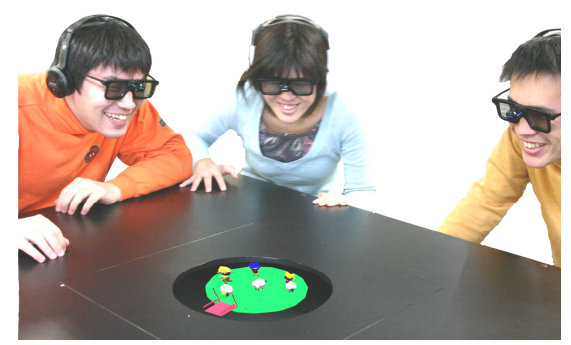

Fig. 4. Snapshot of users enjoying Baa Baa White Sheep

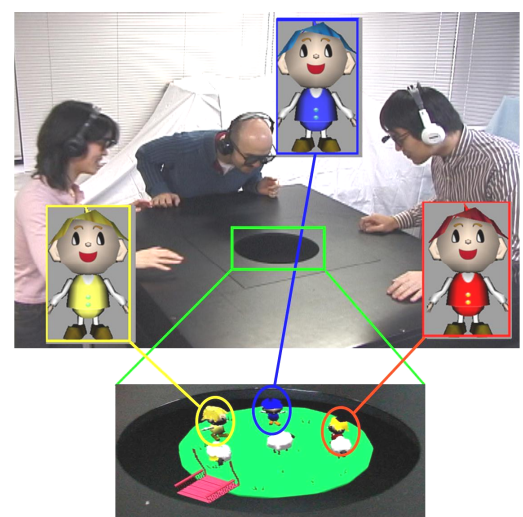

Fig. 5. Correspondence between users and characters

controllers or mice. In the scenario of this application, users have to cooperatively drive sheep into the fold taking into consideration the relative positions of other users, while the sheep tries to escape from the users. Figure 5 shows the correspondence between users and characters. If a user starts moving, the system detects his/her motion and changes the animation of the corresponding character to "walking" from "standing." At the same time, the user can hear the sound of footsteps fitting the "walking" animations. Moreover, the other users can also hear the sounds of footsteps according to the relative position with the user. And if a user comes closer to the sheep, only he/she hears the sound of the bleat. In this way, users enjoy interactions with a virtual world using physical movements and the relative positions to others, while the user feels the character as another human being. The video figure shows details of this application.

\section{Conclusions}

In this paper, we proposed a system for multiple co-located users to enjoy interactive multimedia contents while sharing the same time and space. We described the design approach and implementation of the system followed by two application examples. In the future, we are planning to explore new content suitable for stereoscopic animation with individual sound using physical body gestures or utterances as well as using physical movements and relative positions to others. Moreover, we also plan to look into studying how users perform and cooperate with a variety of personalities and leadership qualities in these environments.

\section{Acknowledgments}

This study was supported in part by "Global COE (Centers of Excellence) Program" of the Ministry of Education, Culture, Sports, Science and Technology, Japan. 


\section{References}

1. Agrawala, M., Beers, A.C., Frohlich, B., Hanrahan, P.: The two-user responsive workbench: support for collaboration through individual views of a shared space. In: Proc. of SIGGRAPH, pp. 327-332 (1997)

2. Bimber, O., Frohlich, B., Schmalstieg, D., Encarnacao, L.M.: The virtual showcase. IEEE Computer Graphics and Applications 21(6), 48-55 (2001)

3. Blaine, T., Perkis, T.: The Jam-O-Drum interactive music system: a study in interaction design. In: Proc. of the 3rd Conference on Designing Interactive Systems, pp. 165-173 (2000)

4. Endo, T., Kajiki, Y., Honda, T., Sato, M.: Cylindrical 3D display observable from all directions. In: Proc. of the 8th Pacific Conference on Computer Graphics and Applications, pp. 300-306 (2000)

5. Favalora, G., Dorval, R.K., Hall, D.M., Giovinco, M., Napoli, J.: Volumetric threedimensional display system with rasterization hardware. In: Proc. of SPIE, vol. 4297, pp. 227-235 (2001)

6. Jordà, S., Geiger, G., Alonso, A., Kaltenbrunner, M.: The reacTable: exploring the synergy between live music performance and tabletop tangible interfaces. In: Proc. of the 1st International Conference on Tangible and Embedded Interaction, pp. 139-146 (2007)

7. Kitamura, Y., Nakayama, T., Nakashima, T., Yamamoto, S.: The IllusionHole with polarization filters. In: Proc. of ACM Symposium on Virtual Reality Software and Technology, pp. 244-251 (2006)

8. Kusunoki, F., Eguchi Yairi, I., Nishimura, T.: Multi-Audible table for collaborative work. In: Proc. of ACM SIGCHI International Conference on Advances in computer entertainment technology, pp. 67-73 (2004)

9. Morris, M.R., Morris, D., Winograd, T.: Individual audio channels with single display groupware: effects on communication and task strategy. In: Proc. of ACM Conference on Computer Supported Cooperative Work, pp. 242-251 (2004)

10. Müller-Tomfelde, C., Steiner, S.: Audio-enhanced collaboration at an interactive electronic whiteboard. In: Proc. of the 2001 International Conference on Auditory Display, pp. 267$271(2001)$

11. Otsuka, R., Hoshino, T., Horry, Y.: Transpost: all-around display system for 3D solid image. In: Proc. of ACM Symposium on Virtual Reality Software and Technology, pp. 187194 (2004)

12. Patten, J., Recht, B., Ishii, H.: Audiopad: a tag-based interface for musical performance. In: Proc. of Conference on New Interface for Musical Expression, pp. 24-26 (2002) 